\title{
Energy Consumption Scheduling in a Smart Grid Including Renewable Energy
}

\author{
Nadia Boumkheld*,**, Mounir Ghogho*****, and Mohammed El Koutbi*
}

\begin{abstract}
Smart grids propose new solutions for electricity consumers as a means to help them use energy in an efficient way. In this paper, we consider the demand-side management issue that exists for a group of consumers (houses) that are equipped with renewable energy (wind turbines) and storage units (battery), and we try to find the optimal scheduling for their home appliances, in order to reduce their electricity bills. Our simulation results prove the effectiveness of our approach, as they show a significant reduction in electricity costs when using renewable energy and battery storage.
\end{abstract}

Keywords

Demand Response, Energy Management, Energy Scheduling, Optimization

\section{Introduction}

The term 'smart grid' refers to the use of computers, communication, sensing, and control technology that operates in parallel with the electrical grid in order to achieve the following goals:1) enhance the reliability of the delivery of electrical power by increasing user visibility and participation; 2) enable new products, services, and markets, and facilitate the interconnection of new generation sources to the grid; 3) reduce carbon emissions and preserve a clean and healthy environment by including renewable energy in the grid; and 4) include the deployment of demand response (DR) programs that refer to mechanisms that encourage consumers to reduce their peak demand for electricity, and as a result lower their electricity costs.

The smart grid aims to provide a high quality and well-managed power grid service while reducing energy wastage in production and transport [1]. To achieve this, future power grids need to motivate the active participation of consumers, and also enable the inclusion of renewable energy sources, distributed storage, and DR programs. Numerous studies have been carried out in the scheduling and optimization of energy consumption. In [2] the authors worked on a micro-grid with distributed resources, and implemented an optimal scheduling of the energy consumed in smart homes by using mixed integer linear programming. Tasks were scheduled based on their given operation time window

\footnotetext{
* This is an Open Access article distributed under the terms of the Creative Commons Attribution Non-Commercial License (http://creativecommons.org/licenses/by-nc/3.0/) which permits unrestricted non-commercial use, distribution, and reproduction in any medium, provided the original work is properly cited. Manuscript received October 10, 2013; accepted December 4, 2013; onlinefirst January 28, 2015. Corresponding Author: Mohammed El Koutbi (koutbm@gmail.com)

* ENSIAS-University Mohamed V Rabat, Morocco (nadiabo8891@gmail.com, koutbm@gmail.com)

** Information and Communication Technologies Laboratory, International University of Rabat, Rabat, Morocco (nadiabo8891@gmail.com, mounir.ghogho@uir.ac.ma)

*** The School of Electronic and Electrical Engineering, University of Leeds, Leeds, UK (m.ghogho@leeds.ac.uk)
} 
(earliest starting time and latest ending time), with the objective of minimizing the daily power costs, and shaving back the power consumption peak. In [3] the authors dealt with the optimization of house energy management, which plays a key role in improving the efficiency of the entire grid network. Residential houses were equipped with photovoltaic (PV) panels and batteries for energy storage. Optimization models were proposed to allow for the managing of the daily energy load for single and multi-user cases, in order to reduce users' energy bills and to improve the overall energy system's performance. The proposed methods scheduled energy tasks by defining the energy plan for the next day. For example:

- When to buy, sell, and store energy

- When to start home appliances.

In order to define the energy plan for the next day, the models proposed require predictions on PV panels, and the future usage of devices. With the development of DR programs in smart grids, the authors in [1] presented a solution for the demand-side energy management problem. Which was considered to be the scheduling of a consumer's daily tasks according to real-time pricing of the market and the user's specified deadlines. The corresponding optimization problem is NP-hard, so they proposed a min-max scheduling algorithm, which achieves significant peak-shaving, cost reduction, and risk aversion for the consumers. In [4], the authors implemented a demand-side management (DSM) program that focuses on interactions between the users in order to coordinate their usage, to reduce the peak-to-average ratio (PAR) in the load demand, and to minimize their energy costs. As such, they proposed an incentive-based consumption-scheduling scheme. They considered a scenario where many customers share a source of energy (i.e., a generator) and each customer is equipped with an energy consumption scheduler (ECS), which is deployed inside a smart meter. Smart meters with ECS functionality interact by running a distributed algorithm to find the optimal energy consumption schedule for each user in the network.

In this work, we consider the same approach used in [4], where multiple users share a source of energy (utility), and a distributed algorithm is applied to find the best schedule for each user. However, we extend this existing work by including renewable energy (wind turbines) and electricity storage (battery), which will be shown to achieve more reduction in users' electricity costs.

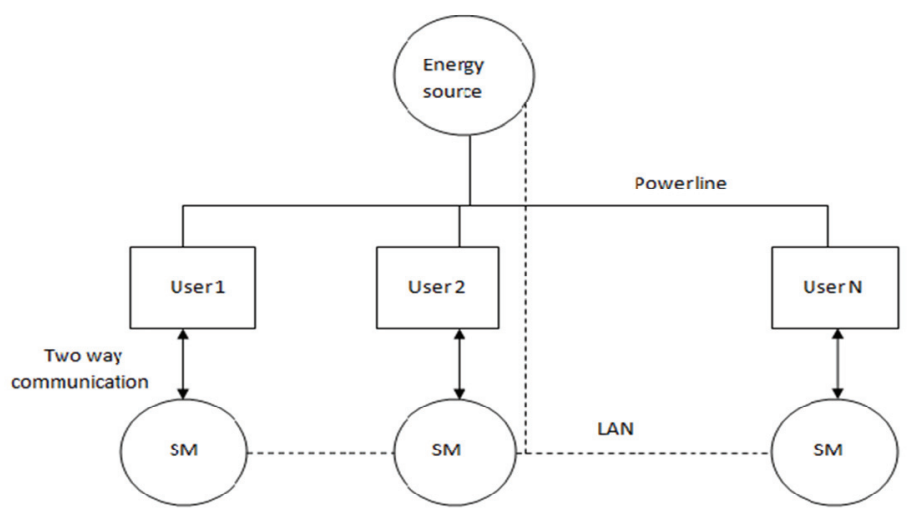

Fig. 1. The smart grid system. 
In Section 2, we will describe briefly the previous work done on this topic. In Section 3 we present our proposed extension. In Section 4, we present our simulation results to illustrate the improvements achieved by the proposed method. Finally, in Section 5, we will present our conclusions.

\section{Brief Description of Previous Work}

The system considered in [4] is described in Fig. 1. The authors considered a group of customers where each one was equipped with a smart meter with an ECS for the scheduling of the different home appliances. Smart meters are connected to each other and to the utility through a Local Area Network (LAN). They formulated a distributed algorithm to be run in each ECS unit, in order to determine the best schedule for every user that will allow him/her to reduce his/her electricity costs. In other words, each user solves an energy cost minimization problem and broadcasts his/her scheduling vector to the other ECS units. This way, the users cooperate in order to find their best schedule and pay less, and this will also achieve the best performance for the entire system.

In the next section, our objective is to introduce renewable energy (wind) and battery storage, to reduce users' energy costs by taking advantage of free wind energy and storage capabilities that will allow users to store energy when there is a surplus, and use it during peak hours.

\section{Integrating Renewable Energy in Energy Consumption Scheduling}

We are proposing the adding of renewable energy (wind turbine) and electricity storage (battery) to the grid, so as to take advantage of free natural energy and to also save electricity for later usage, and as a result to have more reductions in energy consumption costs for the consumers. In this section we will first formulate the optimization problem, and then we will show the results and compare them with those from previous work.

In our work, a user has many appliances. We considered shiftable household appliances (television, washing machine, etc.) where the user sets the time intervals (start and end time) of each appliance [4]. Also, for each appliance we have a predetermined daily consumption amount [4] (Note: when scheduling the power consumption of the appliance, the total power consumed during the assigned time interval should be equal to the predetermined energy). In addition to this, each appliance had a minimum standby power level and a maximum power level [4].

We considered the following two scenarios: the energy optimization for a single house (user) and the energy optimization for many houses (users).

\subsection{Single User}

In this case, the user (a house) was equipped with a wind turbine and electrical storage (battery), and had a grid connection that allowed him/her to buy electricity from the grid. Our task consisted of scheduling the power consumption of the different home appliances of the user, in order to minimize the electricity costs, during one day ( 24 hours). Our optimization is subject to many constraints, as described below. 


\subsubsection{Storage constraints}

- The power stored at hour $h$ depends on the power stored at $h-1$ and on the charge and discharge rates of the battery [2]:

$$
E_{\text {bat }}^{h}=E_{b a t}^{h-1}+C_{r}^{h} \eta-D_{r}^{h} / \eta
$$

$E_{\text {bat }}^{h}: \quad$ Energy stored in the battery at hour $h$.

$E_{\text {bat }}^{h-1}$ : Energy stored in the battery at hour $h-1$.

$C_{r}^{h} \quad$ : Charge rate at hour $h$.

$\eta \quad$ : Electricity chargeldischarge efficiency.

$D_{r}^{h} \quad$ : Discharge rate at hour $h$.

- For the battery, the energy level is bounded between the battery capacity and the minimum energy level, so as not to damage the battery [5].

$$
E_{\min } \leq E_{\text {bat }}^{h} \leq E_{\max }
$$

- The charge and discharge rates of electricity can not exceed the electricity charge and discharge limits [2]:

$$
C_{r}^{h} \leq C_{L}^{h}, D_{r}^{h} \leq D_{L}^{h}
$$

$\mathrm{C}_{\mathrm{L}}^{\mathrm{h}}$ : Charge limit.

$\mathrm{D}_{\mathrm{L}}^{\mathrm{h}}$ : Discharge limit.

- In addition we have:

$$
C_{r}^{h} \geq 0, D_{r}^{h} \geq 0, E_{\text {in }}^{h} \geq 0
$$

where $E_{i n}^{h}$ denotes the energy bought from the grid at each hour h.

\subsubsection{Energy balance}

The electricity consumed at each time consists of the energy supplied by the wind, plus the electricity received from storage and the grid, minus the electricity sent to the storage, i.e.

$$
E_{c}^{h}=E_{\text {wind }}^{h}+E_{i n}^{h}+D_{r}^{h}-C_{r}^{h}
$$


$E_{c}^{h}:$ The energy consumed at each hour $h$.

$E_{\text {wind }}^{h}$ : The energy coming from the wind at each hour $h$.

\subsubsection{Optimization problem}

Our goal is to minimize the cost of electricity purchased from the grid. We assumed a constant cost for the maintenance of the wind and battery. As such, this cost is independent of the schedule. Therefore, the optimization problem is stated as:

$$
\min _{E_{\text {bat }}^{h}, C_{r}^{h}, D_{r}^{h}, E_{\text {in }}^{h}, E_{c}^{h}} \sum_{h \in H} C_{h}\left(E_{\text {in }}^{h}\right) \operatorname{s.t}(1),(2),(3),(4),(5)
$$

$C_{h}\left(L_{h}\right)$ is cost function for the energy purchased from the grid; $C_{h}(L)=a_{h} L^{2}$ was used in [4], where $a_{h}$ is the price at hour $h$; there are two prices: daytime price, $a_{D},(h \in[1 \ldots 16])$ and nighttime price, $a_{N}$, $(h \in[17 \ldots 24])$.

\subsection{Multiple Users}

Here we considered that there were many users where each one was equipped with a wind turbine and a battery, and we minimized the total cost for all users. The problem is stated as:

$$
\min \sum_{n \in N} \sum_{h \in H} C_{h}\left(E_{i n, n}^{h}\right)
$$

subject to the constraints (1)-(5) for each user.

\section{Simulation Results}

Our simulations results were obtained using MATLAB and YALMIP, which is a free MATLAB toolbox for convex and non-convex optimization problems.

\subsection{Single User}

We first took the case of one user (one house) that was equipped with four shiftable appliances; where each appliance must work during a time interval:

- Appliance 1: from $h=13$ to $h=15$.

- Appliance 2: from $h=7$ to $h=12$.

- Appliance 3: from $h=12$ to $h=15$.

- Appliance 4: from $h=4$ to $h=10$.

The house was equipped with a wind turbine and energy storage with the following parameters: the 
minimum energy level was $2 \mathrm{kWh}_{\mathrm{e}}$, the battery capacity was $20 \mathrm{kWh}_{\mathrm{e}}$, the charge and discharge efficiency was 0.95 , the charge and discharge limits were equal to $20 \mathrm{~kW}_{\mathrm{e}}$, the day time price was $a_{D}=0.3$ cents, and the nighttime price was $a_{N}=0.2$ cents.

In order to see the difference between ECS scheduling with and without the use of renewable energy and battery storage, we plotted the energy consumption and the energy cost in these two cases, which we show in Fig. 2 and 3.

From Figs. 2 and 3, we can see that the peak energy cost with ECS scheduling is equal to 4.8 USD, and is reduced to 2.56 USD when we add renewable energy and battery storage.

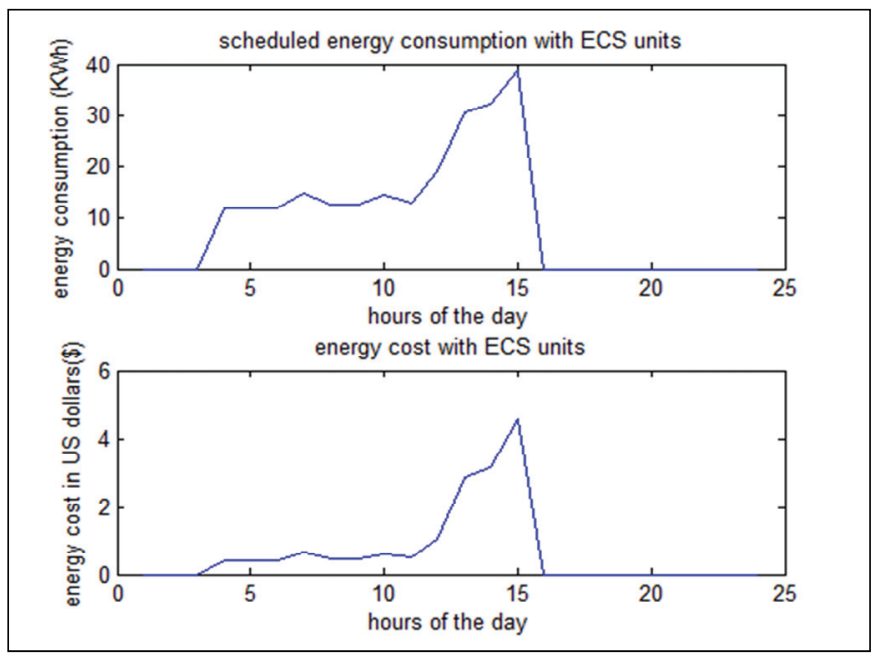

Fig. 2. Energy consumption and costs for one user using energy consumption scheduler (ECS) scheduling.

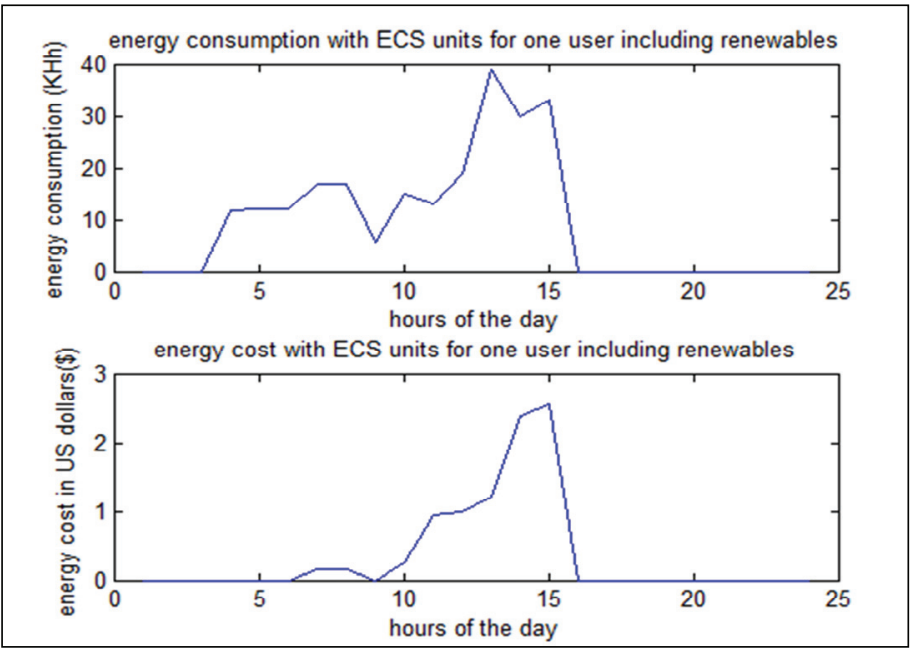

Fig. 3. Energy consumption and costs for one user using energy consumption scheduler (ECS) scheduling with wind energy and battery storage. 


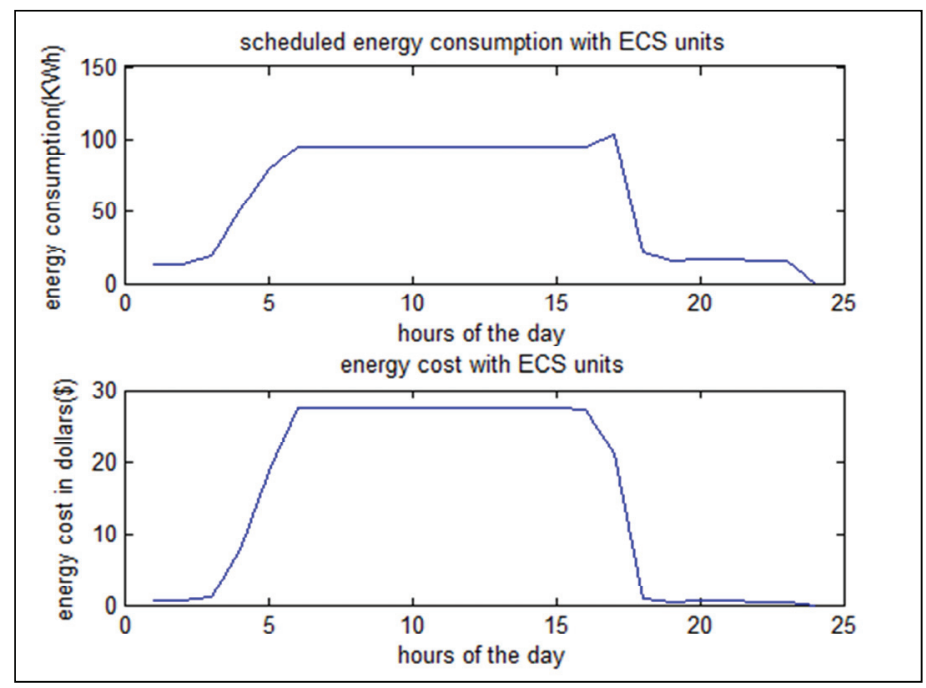

Fig. 4. Energy consumption and costs for the multiuser case when using energy consumption scheduler (ECS) scheduling.

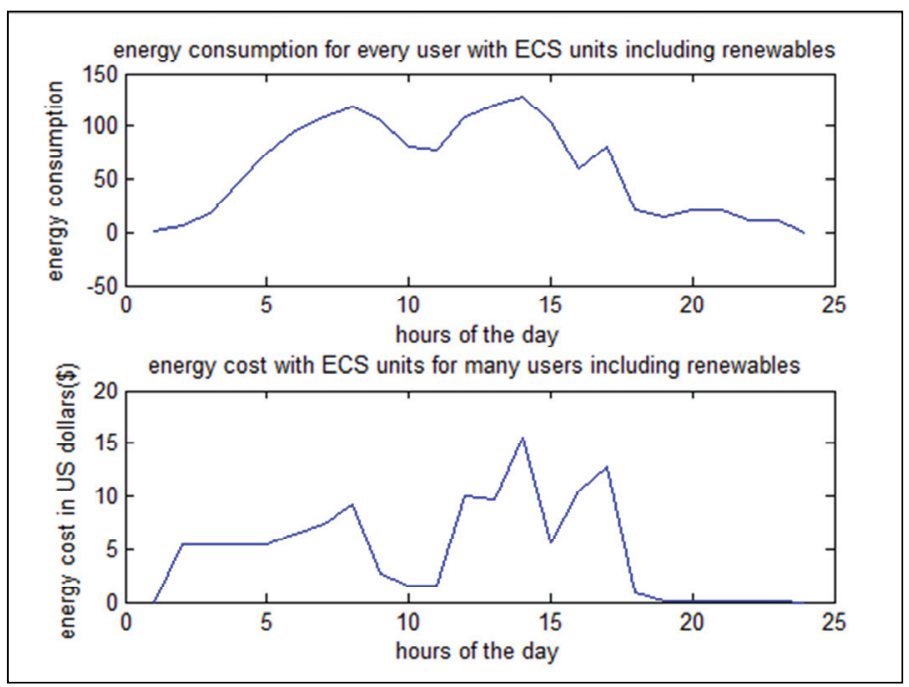

Fig. 5. Energy consumption and cost for the multiuser case when using energy consumption scheduler (ECS) scheduling with wind energy and battery storage.

\subsection{Multiple Users}

We used 7 houses. Each house had 4 shiftable appliances and was equipped with a wind turbine and a battery. The characteristics of the battery were the same as in the single user case, except for its capacity and charge and discharge limits, which were taken to be $50 \mathrm{kWh}_{\mathrm{e}}$. The daytime and nighttime prices of electricity were the same as in the single user case.

Fig. 4 shows the energy consumption and cost using the ECS scheduling (as in [4]). Fig. 5 depicts the energy consumption and cost when we added the renewable energy and the battery. We can see that the 
cost is reduced. More specifically, when we used ECS without renewable energy and a battery, the total cost was 353.84 USD, but when we added renewable energy and the battery, the total cost was reduced to 115.93 USD. However, we did not achieve any improvement in the PAR.

\section{Conclusion}

In this paper, we first reviewed the ECS scheduling that was proposed by the authors in [4]. Then, we extended their work to include renewable energy (wind) and electricity storage or battery to save electricity when there is a surplus in energy production. Even though we did not achieve a reduction in the PAR, the total electricity cost was significantly reduced.

\section{References}

[1] J. Xiao, J. Y. Chung, J. Li, R. Boutaba, and J. K. Hong, "Near optimal demand-side energy management under real-time demand-response pricing," in Proceedings of the International Conference on Network and Service Management (CNSM2010), Ontario, Canada, 2010, pp. 527-532.

[2] D. Zhang, L. G. Papageorgiou, N. J. Samsatli, and N. Shah, "Optimal scheduling of smart homes energy consumption with microgrid," in Proceedings of the 1st International Conference on Smart Grids, Green Communications and IT Energy-aware Technologies (ENERGY2011), Venice, Italy, 2011, pp. 70-75.

[3] A. Barbato, A. Capone, G. Carello, M. Delfanti, M. Merlo, and A. Zaminga, "Cooperative and non-cooperative house energy optimization in a smart grid perspective," in Proceedings of the IEEE International Symposium on a World of Wireless, Mobile and Multimedia Networks (WoWMoM2011), Lucca, Italy, 2011, pp. 1-6.

[4] A. H. Mohsenian-Rad, V. W. Wong, J. Jatskevich, R. Schober, and A. Leon-Garcia, "Autonomous demand-side management based on game-theoretic energy consumption scheduling for the future smart grid," IEEE Transactions on Smart Grid, vol. 1, no. 3, pp. 320-331, 2010.

[5] A. Barbato, A. Capone, G. Carello, M. Delfanti, M. Merlo, and A. Zaminga, "House energy demand optimization in single and multi-user scenarios," in Proceedings of the IEEE International Conference on Smart Grid Communications (SmartGridComm2011), Brussels, Belgium, 2011, pp. 345-350.

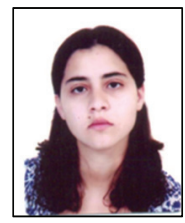

Nadia Boumkheld http://orcid.org/0000-0002-5058-4847

She Received the Master degree in 2010 in Computer Science and telecommunication from the Faculty of Science of Rabat, Morocco. She is currently a Ph.D. student at Université Internationale de Rabat (UIR), and Ecole Nationale Supérieure d'Informatique Et Analyse Des Systèmes (ENSIAS). Her research interests include smart grids and wireless communication networks.

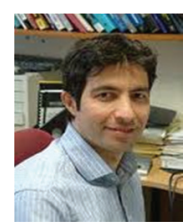

\section{Mounir Ghogho}

Mounir Ghogho received the M.Sc. degree in 1993 and the Ph.D. degree in 1997 from the National Polytechnic Institute of Toulouse, France. He was an EPSRC Research Fellow with the University of Strathclyde, Glasgow (Scotland), from September 1997 to November 2001. Since December 2001, he has been a faculty member with the 
school of Electronic and Electrical Engineering at the University of Leeds (UK), where he currently holds a Chair in Signal Processing and Communications. In January 2010, he joined the International University of Rabat (Morocco) where he is currently the Director of ICT Research. He is currently an Associate Editor of the IEEE Signal Processing magazine. He served as an Associate Editor of the IEEE Signal Processing Letters from 2001 to 2004, the IEEE Transactions on Signal Processing from 2005 to 2008, and the Elsevier Digital Signal Processing journal from 2011 to 2012. He served as a member of the IEEE Signal Processing Society SPCOM Technical Committee from 2005 to 2010, a member of IEEE Signal Processing Society SPTM Technical Committee from 2006 to 2011, and is currently a member of the IEEE Signal Processing Society SAM Technical Committee. He was the general cochair of the eleventh IEEE workshop on Signal Processing for Advanced Wireless Communications (SPAWC'2010) and the $21^{\text {st }}$ edition of the European Signal Processing conference (EUSIPCO2013), the technical co-chair of the MIMO symposium of IWCMC 2007 and IWCMC 2008, and a technical area co-chair of Eusipco 2008, Eusipco 2009 and ISCCSP'05. His research interests are in signal processing and its applications (wireless communication, intelligent transport, smart grid, etc.). He has published around 210 journal and conference papers. He has supervised more than $25 \mathrm{PhD}$ students. He held invited scientist/ professor positions at many institutions including the US Army Research Lab (USA), Télécom ParisTech (France), National Institute of Informatics (Japan), University Carlos 3rd of Madrid (Spain), ENSICA (France), Darmstadt Technical University (Germany), Minnesota University (USA), Beijing University of Posts and Telecommunication (China), and University Mohamed V (Morocco). Prof. Ghogho was awarded the prestigious five-year UK Royal Academy of Engineering Research Fellowship in September 2000. He is one of the recipients of the highly and internationally competitive IBM Faculty award in 2013.

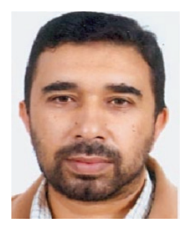

\section{Mohammed El Koutbi}

He was born in 1965 in Rabat, Morocco. He obtained his engineering degree in computer science from 'Ecole Mohammadia d'ingénieurs' (engineering school) in 1988. He obtained his Ph.D. in computer science from Montreal University, Canada in July 2000. He works now as a full professor at ENSIAS, Mohammed V University Souissi, Rabat, Morocco. He is very active in the domain of ad hoc routing protocols. $\mathrm{He}$ also does some research in the field of model transformations based on UML. He's currently the responsible of the MIS (Mobile Intelligent Systems) research team. 